\title{
Rhinosinusal Inflammation and High Mobility Group Box 1 Protein: A New Target for Therapy
}

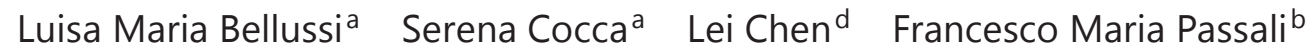 \\ Condrut Sarafoleanu ${ }^{c}$ Desiderio Passali ${ }^{a}$ \\ aENT Section, Department of Medical-Surgical Sciences and Neurosciences, University of \\ Siena, Siena, and ${ }^{b}$ ENT Clinic, Tor Vergata University, Rome, Italy; ${ }^{c}$ ENT Department,

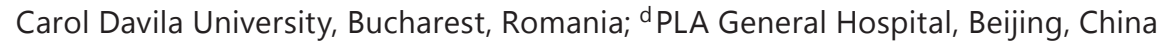

\section{Key Words}

Rhinosinusal inflammation · High mobility group box 1 protein · Glycyrrhetic acid

\begin{abstract}
Background/Aims: High mobility group box 1 (HMGB1) is a protein belonging to the class of damage-associated molecular pattern molecules, which activates innate immunity and powerful inflammatory factors. The aim of this review is to show the importance of HMGB1 in the pathogenesis of nasal inflammatory diseases and to suggest that inhibition of HMGB1 may be an innovative therapeutic target. Methods: We used immunohistochemistry to study whether HMGB1 increases in chronic rhinosinusitis with nasal polyps and whether its expression is associated with eosinophils and inflammatory cytokines. Using primary cultures of human nasal epithelial cells, we localised lipopolysaccharide-induced active translocation and release of HMGB1 by immunofluorescence assay and Western blot. Results: Patients with severe symptoms have the highest HMGB1 serum levels. Glycyrrhetic acid inhibits the chemotactic and mitogenic function of HMGB1, binding to the hydrophobic residues that delimit the pockets in box A and B. Conclusions: Chronic inflammatory diseases of the nose and paranasal sinuses are increasingly prevalent and are a financial burden for society. HMGB1 has been shown to play a role in several inflammatory diseases of otolaryngological interest. The inhibition of HMGB1 may be an innovative therapeutic target for patients with chronic upper airway inflammatory diseases having nasal obstruction as a major symptom.
\end{abstract}

(C) 2016 S. Karger AG, Basel 


\section{Introduction}

Concerning the mechanisms underlying chronic inflammatory pathologies of the upper and lower airways [such as allergic rhinitis (AR), chronic rhinosinusitis (CRS) with/without nasal polyps, asthma, chronic obstructive pulmonary disease, and cystic fibrosis], the role of the respiratory epithelium as a barrier against infection and injury has been stressed in recent decades [1].

There are different barrier levels: the mechanical barrier represented by the integrity of epithelial cells and their junctions [2], the physical/chemical barrier (mucociliary transport made up of cilia and mucus, with proteins and enzymes dissolved in it) and the innate immunity barrier. The cells (monocytes/macrophages, APCs, and NK-cells) of innate immunity are activated on recognition of a wide range of antigens and express various pattern recognition receptors for specific molecules on pathogens, known as pathogen-associated molecular patterns (PAMPs) [3]. Toll-like receptors (TLRs) are specific pattern recognition receptors recognizing PAMPs in various cell compartments, and their interaction triggers release of inflammatory cytokines for host defence.

The innate immune system is activated by endogenous danger signals, i.e. endogenous peptides constitutively present in leukocyte granules or epithelial cells and released by damaged and necrotic cells. Together with PAMPs, these peptides constitute the large family of damage-associated molecular patterns and are the first host response to exogenous (infections) and endogenous (injury) danger signals. Responses of the innate immune system are important not only to eliminate pathogens but also to develop pathogen-specific adaptive immunity, which is mediated by B and T cells.

Our recent studies have focused on the interplay between TLRs, PAMPs and the high mobility group box 1 protein (HMGB1), an ancient evolutionary protein belonging to the damage-associated molecular patterns family. Under homeostatic conditions, HMGB1 is a nonhistonic DNA-binding protein [4], but when released extracellularly, it plays the role of a potent pro-inflammatory cytokine [5]. Extracellular HMGB1 binds to different membrane receptors (TLR4, TLR9, and RAGE), and through activation of the transcription factor nuclear factorkappa B, it causes release of pro-inflammatory mediators, cytokines and chemokines, induces endothelial activation, and increases survival of inflammatory cells, mainly eosinophils.

Low levels of HMGB1 usually mediate beneficial responses to environmental or endogenous threats, enhancing the innate and adaptive immune systems and promoting inflammation and tissue repair. High levels cause acute damage: indeed, when HMGB1 binds to receptors expressed by endothelial cells, it promotes inflammatory loops through continuous recruitment of inflammatory cells, mainly eosinophils, prolonging their survival, and release of inflammatory mediators, such as eosinophil cationic protein and major basic protein, with subsequent epithelial barrier damage.

The HMGB1 protein has been shown to play a role in the pathogenesis of inflammatory diseases, such as hepatitis, arthritis, stroke, liver and kidney ischemia, sepsis [6], rheumatoid arthritis [7] and systemic lupus erythematosus [8]. Concerning the airways, increased induced sputum and circulating HMGB1 levels have been found in patients with asthma and chronic obstructive pulmonary disease and have been associated with disease severity, thus sustaining the hypothesis that HMGB1 may play an important role in the development of pulmonary inflammation and lung matrix degradation [9]. The same results have been found in cystic fibrosis patients, where HMGB1 levels are elevated in bronchoalveolar lavage fluid [10] and bacterial clearance of Pseudomonas aeruginosa is low [11].

Thanks to its role in the innate and adaptive immune systems, HMGB1 has been considered a potent antibacterial protein produced and harboured in the adenoid tissue; by delaying or inhibiting bacterial penetration, it may contribute to the barrier defence system of the mucosa of the upper respiratory tract [12]. 
Fig. 1. Box plots of the semi-quantitative analysis of cells staining positive for the HMGB1 protein. The boxes represent the 25th75th percentiles, the line within the boxes, the median, and the lines outside the boxes, the minimum and maximum values. $p$ values were compared between the groups. * $\mathrm{p}<0.05$; ${ }^{* *} \mathrm{p}<0.01$; $* * * \mathrm{p}<0.001$. From Bellussi et al. [14].

Bellussi et al.: Rhinosinusal Inflammation and High Mobility Group Box 1 Protein: A New Target for Therapy

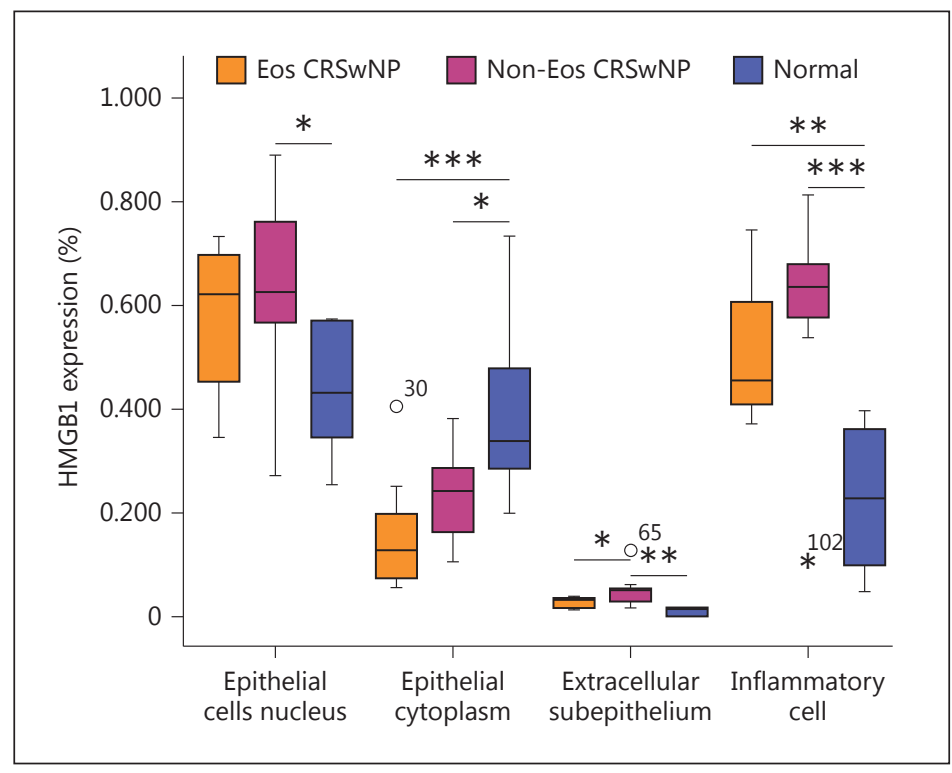

\section{HMGB1 and Rhinosinusitis}

Our recent research was addressed to the role of HMGB1 in the pathogenesis of chronic inflammatory diseases of the nasal mucosa, such as AR, CRS [13] and nasal polyposis [14]. The aims of our first study [14] were: (1) to determine whether HMGB1 increases in CRS with nasal polyps (CRSwNP); (2) to determine whether its expression is associated with eosinophils or with the cytokines TNF- $\alpha$, IL- 5 and IL-8, typically present in chronic inflammation of the nose and paranasal sinuses, and (3) to investigate the hypothesis that this protein plays a role in the pathogenesis of nasal polyposis. For this purpose, nasal polyp tissue from 21 patients with CRSwNP, including 1 patient with asthma and 2 patients with AR and 8 healthy control subjects, was collected. CRSwNP was confirmed by medical history, nasal endoscopy and computed tomography. Control subjects were patients with rhinorrhoea of cerebrospinal fluid, fracture of the optic canal or a benign skull base tumour without a history of sinus disease or asthma. Biopsy specimens of nasal mucosa and nasal polyps were obtained before surgery. Samples were stained with haematoxylin and eosin (HE), and part of the fresh tissue was snap-frozen at $-180^{\circ}$ for immunohistochemical staining. HMGB1 protein expression and its correlation with eosinophils and IL-5, IL-8 and TNF- $\alpha$ cytokines was investigated.

Based on the HE staining, subjects were divided into three groups: 8 control subjects, 10 eosinophilic CRSwNP patients and 11 non-eosinophilic CRSwNP patients. Expression of HMGB1 in inflammatory cells of patients with eosinophilic or non-eosinophilic CRSwNP was significantly greater than in controls. The HMGB1 protein was evident in the nucleus of epithelial cells or as focal subepithelial infiltration and in inflammatory cells of patients, whereas HMGB1 staining was significantly less in epithelial cytoplasm (fig. 1). We did not find any significant difference in HMGB1 expression between eosinophilic CRSwNP and noneosinophilic CRSwNP, suggesting that HMGB1 may play a crucial role in the pathogenesis of CRSwNP, irrespective of aetiological stimuli. These results suggested that eosinophils and IL-5-, IL-8- and TNF- $\alpha$-positive cells may be involved in the regulation of HMGB1.

These findings are confirmed by those of other authors. Hong et al. [15] analysed sinus tissue samples from 10 patients with CRS and 10 controls, i.e. patients undergoing surgery for endoscopic endonasal reconstruction of the orbital wall due to blowout fracture. Samples 
were immunostained for localisation of HMGB1 and image analysis was performed: HMGB1 was more highly expressed in epithelial cells and in inflammatory cells of patients with CRS. Semi-quantitative reverse transcriptase-polymerase chain reaction, real-time polymerase chain reaction and Western blot analysis showed that the expression of the HMGB1 protein was significantly greater in CRS patients than in controls.

The aim of our second study [16] was to confirm the role of HMGB1 in nasal polyposis and CRS and to determine whether its expression and localisation (nuclear, cytoplasmic, extracellular) were related to the severity and complexity of the histological and clinical manifestations. Ten biopsies of nasal mucosa from CRS patients without nasal polyps and 31 CRS patients with nasal polyposis were randomly selected; three biopsies of normal nasal mucosa harvested from healthy patients with no symptoms of CRS or nasal allergies were included as controls. No inflammatory infiltrate, fibrosis, newly formed vessels or eosinophils were found by the pathologist in these specimens. Patient assessment was based on clinical history, symptoms, severity evaluation, presence or absence of allergy, asthma, acetylsalicylic acid intolerance, other allergic manifestations (drug allergy), previous surgery and recurrences.

The tissue samples were tested by immunohistochemistry using HMGB1 polyclonal antibody followed by counterstaining with Mayer's haematoxylin. The biopsy samples were analysed with two different aims. The first aim was to evaluate HMGB1 expression and its cellular distribution. For this purpose, nuclear, cytoplasmic and extracellular staining was estimated as a percentage of total staining in the area in question and the intensity of HMGB1 positivity was expressed as a percentage. The second aim was to compare this distribution with inflammatory infiltrate and clinical severity. As expected, all patients with elevated inflammatory infiltrate and eosinophils had an allergic hyperreactive condition or co-morbidity, which may induce and sustain nasal mucosal inflammation. We investigated the correlation between eosinophils and nuclear HMGB1 values and found low nuclear levels in patients without eosinophils and higher levels in those with higher eosinophil scores.

Since according to our hypothesis, HMGB1 may be a marker of disease activity, we evaluated allergic and non-allergic patients for the protein. We found a statistically significant increase in extracellular $(p=0.0380)$ and nuclear HMGB1 $(p=0.0499)$ in allergic patients. Other allergic hyperactive conditions, such as asthma, intolerance to NSAIDs (non-steroidal anti-inflammatory drugs) and antibiotic allergy, were also assessed in relation to HMGB1 levels. A significant correlation was found between extracellular HMGB1 and NSAID intolerance ( $p=0.0022)$, which confirmed our hypothesis that HMGB1 could be a useful marker of disease activity. The higher expression of extracellular HMGB1 in patients with severe clinical and inflammatory manifestations and associated co-morbidities confirms the role of HMGB1 in induction of the inflammatory reaction and demonstrates its importance for chronic inflammation.

In a cross-sectional study, other authors found that the HMGB1 level in nasal lavage fluid from patients affected by CRS correlated positively with the Lund-Mackay score, thus confirming that the HMGB1 protein secreted into the intercellular spaces of the upper airways should be considered an inflammatory mediator associated with disease severity [17].

Another study [18] by our research group has recently confirmed the hypothesis that up-regulation of HMGB1 could be a significant marker of eosinophilic CRSwNP and contribute to the pathogenesis of CRSwNP along with IL-5, IL-8 and TNF- $\alpha$ (fig. 2).

These results provide interesting insights into inflammatory/anti-inflammatory mechanisms. Pharmacological research could be directed to seek new compounds that decrease extra-cellular levels of the protein by a scavenger mechanism in order to control the inflammatory process without interfering with nuclear transcriptional messengers, at the same time blocking the feedback loop characteristic of the mechanism of action of HMGB1.

To better understand the pathophysiological role of epithelial cells in inflammation, we performed an in vitro experimental study [19] using primary cultures of human nasal 
Fig. 2. Immunohistochemical location of HMGB1 in healthy nasal mucosa and nasal polyps (HE. $\times 400$ ). HMGB1 in the epithelium of a control subject (a), a non-Eos CRSwNP patient (b), an Eos CRSwNP patient (c), and in submucosal tissue of a non-Eos CRSwNP patient (d). HMGB1 expression (brown) in epithelial cells, in infiltrating inflammatory cells, and among infiltrating inflammatory cells. Eos = Eosinophilic. From Chen et al. [17].

Bellussi et al.: Rhinosinusal Inflammation and High Mobility Group Box 1 Protein: A New Target for Therapy

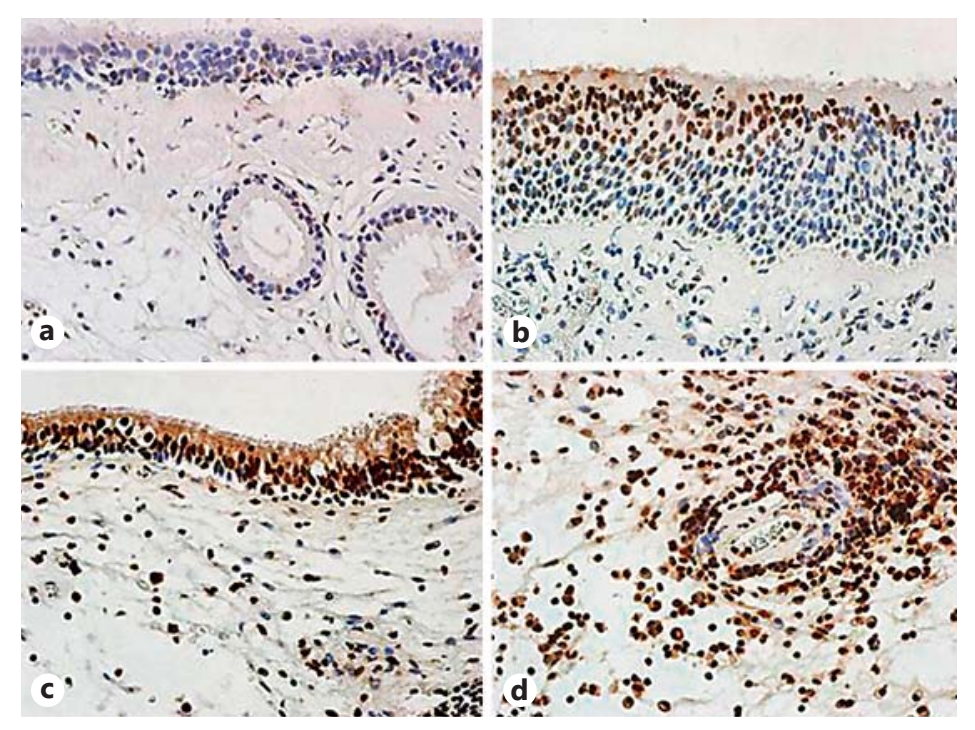

epithelial (HNE) cells, following lipopolysaccharide (LPS)-induced active translocation and release of HMGB1 by immunofluorescence assay and Western blot. We obtained epithelial cells of nasal polyps and paranasal sinus mucosa from 10 patients requiring surgery for sinusitis, excluding cases with non-invasive fungal sinusitis, chronic obstructive pulmonary disease, cystic fibrosis, primary ciliary dyskinesia and severe asthma. Escherichia coli LPS was added during mucociliary differentiation at working concentrations of 10,50 and $100 \mu \mathrm{g} / \mathrm{ml}$. HMGB1 protein levels in epithelial cells and supernatants were assessed by Western blot after $0,12,24,48$ and $72 \mathrm{~h}$.

For the first time, the results documented HMGB1 immunostaining in the nuclei of HNE cells from nasal polyps, confirming our hypothesis that HNE cells are active participants in innate immunity mechanisms. After LPS stimulation, HMGB1 translocated from nuclei to cytoplasm, suggesting that HNE cells are a potential source of the form of HMGB1 secreted in the airways.

This finding was in line with the results of the Western blot analysis; indeed, the level of intracellular HMGB1 did not change in the early stages (0-24 h) of stimulation, but increased significantly at $48 \mathrm{~h}$ and maintained a high level for up to $72 \mathrm{~h}$ (fig. 3). At the same time, secreted HMGB1 was detected in the culture supernatant of LPS-treated HNE cells after $12 \mathrm{~h}$, and the concentration of HMGB1 significantly increased $24 \mathrm{~h}$ after treatment onset and steadily continued to increase.

As a whole, our findings suggest that HMGB1 plays an important role in the induction and prolongation of inflammatory processes of the nose and paranasal sinuses. Understanding the mechanisms of infection- and injury-elicited inflammatory response could be useful for identifying new targets for therapy.

\section{HMGB1 - A New Target for Therapy}

All our studies suggested that HMGB1 inhibition might be an effective and innovative therapeutic target for patients with chronic upper airway and inflammatory diseases of the nasal mucosa [20]. 
Fig. 3. HMGB1 levels released from HNE cells. After exposure to $100 \mu \mathrm{g} / \mathrm{ml}$ of LPS for 0-72 h, HNE cells released variable levels of HMGB1 (Western blot analysis). * $\mathrm{p}<0.05$; ** $\mathrm{p}<0.01$; *** $\mathrm{p}<$ 0.001 . From Chen et al. [18].

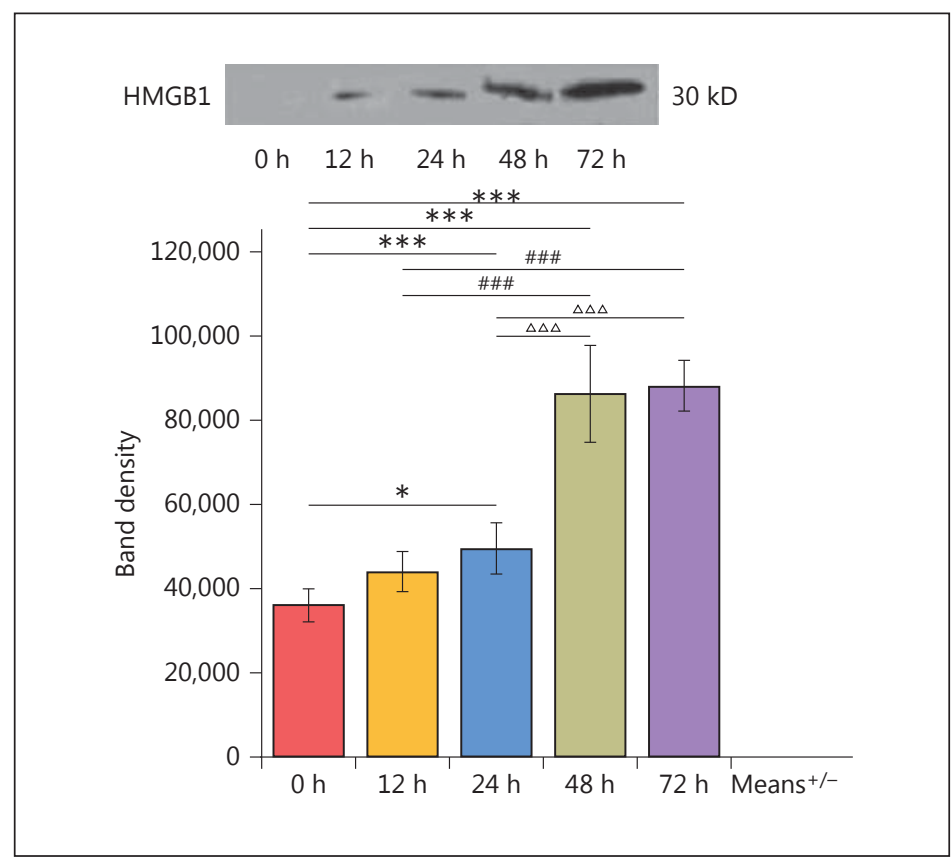

HMGB1 inhibition may be obtained in three ways:

1 blocking HMGB1 release by necrotic cells and activated immune cells; which, however, suppresses the protein's physiological homeostatic function in all cells, with serious consequences;

2 blocking RAGE, TLR2 and TLR9 receptors with antagonist drugs; which, however, is risky because HMGB1 receptors also mediate other immune effects, such as antibacterial activity, and

3 inactivating HMGB1 after its release: this is currently the most effective and safest strategy.

Among molecules that bind HMGB1, glycyrrhizin is a glycoside alkaloid found in large quantities in Glycyrrhiza glabra roots, the plant from which liquorice is extracted [21]. Glycyrrhizin is composed of a molecule of glycyrrhetic acid (GA), the active component of the molecule, and two molecules of glucuronic acid. GA inhibits the chemotactic and mitogenic function of HMGB1, binding to the hydrophobic residues that delimit the pockets in box A and B, without significantly distorting their secondary structure and hence without impeding the ability of DNA binding [22]. GA appears to be well tolerated, and i.v. administration of $240 \mathrm{mg}$ three times a week for 4 weeks has no side effects in humans. Several studies have shown an absence of cytotoxicity even at high concentrations and good pharmacological tolerance of glycyrrhizin by rats and humans [23]. With this background, recent studies have evaluated the potential therapeutic effects of GA in diseases of the nose and paranasal sinuses, particularly CRS with/without nasal polyposis and AR characterised by mucosal inflammation and nasal obstruction.

\section{Conclusions}

Allergic rhinitis (AR), allergen-mediated inflammation of the nasal mucosa that may extend into the paranasal sinuses, is a global health problem, causing major illness and disability worldwide [24]. Its prevalence has significantly increased in the last two decades; 
in many countries, it exceeds $50 \%$ of the population in some age groups. AR is accompanied by a parallel increase in co-morbidities such as asthma, rhinosinusitis and otitis media. Its major symptoms typically include nasal obstruction and rhinorrhoea followed by sneezing with itchy nose, eyes and throat. Nasal congestion is a hallmark of AR and is often the symptom patients find most troublesome and would most like to prevent [25-27]. Typical sleep-related problems seen in AR, such as sleep-disordered breathing, sleep apnoea and snoring, are also associated with nasal obstruction [28]. Children with AR have higher HMGB1 concentrations in sputum, plasma and nasal lavage fluid than non-allergic subjects. Patients with severe symptoms have the highest HMGB1 serum levels. Total IgE serum levels and peripheral eosinophil count are related to visual analogue scale (VAS) values. These outcomes indirectly confirm the relationship between symptom severity assessed by VAS and surrogate markers of allergy severity, such as IgE and eosinophils [29].

Despite the shortage of epidemiological studies exploring the prevalence and incidence of CRS with or without nasal polyps, this condition ranks second among all chronic conditions [30]. In adults, CRS with or without nasal polyps is defined as inflammation of the nose and paranasal sinuses, characterised by two or more symptoms, one of which is either nasal blockage/obstruction/congestion or nasal discharge [31]. Thus, nasal congestion, i.e. a feeling of blockage, fullness or obstruction of the nasal cavity, is a cardinal symptom of common upper respiratory tract inflammatory disorders, including AR and acute and CRS with or without nasal polyposis. It has a complex pathophysiology, involving several inflammatory cytokines that act on neural and vascular structures, leading to dilation of nasal blood vessels, increased secretion, tissue swelling/oedema and ultimately nasal obstruction.

Although various pharmacotherapeutic options to treat nasal obstruction exist, no agent is universally effective, and there is paucity of data supporting commonly used symptomatic therapies, especially in children [32]. Nasal decongestants effectively reduce nasal obstruction [33]. Despite their widespread use, local and systemic side effects are frequent, and it is advisable to limit their period of use by adults and otherwise healthy patients. Topical nasal corticosteroids are commonly prescribed by paediatricians, rhinologists and allergists. They act directly on the nasal mucosa, but systemic dose-related side effects, such as suppression of adrenocortical function, growth, and bone metabolism [34], associated with long-term treatments, can be a problem in children.

The short- and long-term efficacy of a new medical device for treating nasal congestion has recently been demonstrated $[35,36]$. The device acts osmotically and relies on the antiedematous and anti-inflammatory characteristics of components such as eucalyptol, glycyrrhizin and mannitol. Patients' perception of nasal symptoms and objective testing of nasal obstruction were both assessed. Together with subjective self-assessment of the sensation of nasal obstruction by VAS, physical examination of treated patients also showed a significant improvement in turbinate hypertrophy and secretory activity. No adverse effects were reported by the patients over the treatment period and compliance was generally high. In another study [37], the efficacy and safety of GA versus intranasal corticosteroids in the management of symptoms of AR were tested in a paediatric population over a 30-day period. A significant improvement in all major symptoms, including rhinorrhoea, sneezing and nasal obstruction, was found in both groups after treatment, without any significant difference in terms of percentage reaction, but with a better safety profile in the group treated with the medical device. The use of osmotic devices proves to be an efficient solution in the treatment of nasal congestion and helps to clear the nose. Thus, association of GA with osmotic formulations increases the beneficial effects and rapidly reduces oedema, while glycyrrhizin exerts its anti-inflammatory properties.

Clinical and experimental research on these topics should continue, as the results promise to reduce issues which are not only pertinent to the ENT field. 
Bellussi et al.: Rhinosinusal Inflammation and High Mobility Group Box 1 Protein: A New Target for Therapy

\section{Disclosure Statement}

The authors declare that there is no conflict of interest regarding the publication of this paper.

\section{References}

1 Swindle EJ, Collins JE, Davies DE: Breakdown in epithelial barrier function in patients with asthma: identification of novel therapeutic approaches. J Allergy Clin Immunol 2009;124:23-34.

2 Weidinger S, O'Sullivan M, Illig T, Baurecht H, Depner M, Rodriguez E, et al: Filaggrin mutations, atopic eczema, hay fever, and asthma in children. J Allergy Clin Immunol 2008;121:1203-1209.

3 Vroling AB, Fokkens WJ, van Drunen CM: How epithelial cells detect danger: aiding the immune response. Allergy 2008;63:1110-1123.

4 Verrijdt G, Haelens A, Schoenmakers E, Rombauts W, Claessens F: Comparative analysis of the influence of the high-mobility group box 1 protein on DNA binding and transcriptional activation by the androgen, glucocorticoid, progesterone and mineralocorticoid receptors. Biochem J 2002;361:97-103.

5 Scaffidi P, Misteli B, Bianchi ME: Release of chromatin protein HMGB1 by necrotic cells triggers inflammation. Nature 2002;418:191-195.

6 Wang H, Bloom O, Zhang M, Vishnubhakat JM, Ombrellino M, Che J, et al: HMG-1 as a late mediator of endotoxin lethality in mice. Science 1999;285:248-251.

7 Wittemann B, Neuer G, Michels H, Truckenbrodt H, Bautz F: Autoantibodies to nonhistone chromosomal protein HMG-1 and HMG-2 in sera of patients with juvenile rheumatoid arthritis. Arthritis Rheum 1990;33: 1378-1383.

8 Ayer LM, Rubin RL, Dixon GH, Fritzler MJ: Antibodies to HMG proteins in patients with drug-induced autoimmunity. Arthritis Rheum 1994;37:98-103.

9 Hou C, Zhao H, Liu L, Li W, Zhou X, Lv Y, et al: High mobility group protein B1 (HMGB1) in asthma: comparison of patients with chronic obstructive pulmonary disease and healthy controls. Mol Med 2011;17:807-815.

10 Rowe SM, Jackson PL, Liu G, Hardison M, Livraghi A, Solomon GM, et al: Potential role of high-mobility group box 1 in cystic fibrosis airway disease. Am J Respir Crit Care Med 2008;178:822-831.

11 Entezari M, Weiss DJ, Sitapara R, Whittaker L, Wargo MJ, Li J, et al: Inhibition of high-mobility group box 1 protein (HMGB1) enhances bacterial clearance and protects against Pseudomonas Aeruginosa pneumonia in cystic fibrosis. Mol Med 2012;18:477-485.

12 Zetterström CK, Bergman T, Rynnel-Dagöö B, Erlandsson Harris H, Soder O, Andersson U, et al: High mobility group box chromosomal protein 1 (HMGB1) is an antibacterial factor produced by the human adenoid. Ped Res 2002;52:148-154.

13 Passali D, Kern E, Chen L, Bellussi LM: High mobility group box 1 (HMGB1): a new protein in the pathogenesis of ENT inflammatory and infectious diseases. Acta Otorhinolaryngol Ital 2012;32:46-47.

14 Bellussi LM, Chen L, Chen D, Passali FM, Passali D: The role of high mobility group box 1 chromosomal protein in the pathogenesis of chronic sinusitis and nasal polyps. Acta Otorhinolaryngol Ital 2012;32:386-392.

15 Hong SM, Cho JS, Um JY, Shin JM, Park IH, Lee SH, et al: Increased expression of high-mobility group protein B1 in chronic rhinosinusitis. Am J Rhinol Allergy 2013;27:278-282.

16 Bellussi LM, Iosif C, Sarafoleanu C, Jianu E, Duda R, Panaitescu E, et al: Are HMGB1 protein expression and secretion markers of upper airways inflammatory diseases? J Biol Regul Homeost Agents 2013;27:791-804.

17 Min HJ, Kim SJ, Kim TH, Chung HJ, Chung HJ, Yoon JH, Kim CH: Level of secreted HMGB1 correlates with severity of inflammation in chronic rhinosinusitis. Laryngoscope 2015;125:225-230.

18 Chen D, Mao M, Bellussi LM, Passali D, Chen L: Increase of high mobility group box chromosomal protein 1 in eosinophilic chronic rhinosinusitis with nasal polyps. Int Forum Allergy Rhinol 2014;4:453-462.

19 Chen D, Bellussi LM, Passali D, Chen L: LPS may enhance expression and release of HMGB1 in human nasal epithelial cells in vitro. Acta Otorhinolaryngol Ital 2013;33:398-404.

20 Yang H, Wang H, Czura CJ, Tracey KJ: HMGB1 as a cytokine and therapeutic target. J Endotoxin Res 2002;8: 469-472.

21 Mollica L, De Marchis F, Spitaleri A, Dallacosta C, Pennacchini D, Zamai M, et al: Glycyrrhizin binds to highmobility group box 1 protein and inhibits its cytokine activities. Chem Biol 2007;14:431-441.

22 Ploeger B, Mensinga T, Sips A, Seinen W, Meulenbelt L, DeJongh J: The pharmacokinetics of glycyrrhizic acid evaluated by physiologically based pharmacokinetic modeling. Drug Metab Rev 2001;33:125-147.

23 van Rossum TG, Vulto AG, Hop WC, Brouwer JT, Niesters HG, Schalm SW: Intravenous glycyrrhizin for the treatment of chronic hepatitis C: a double-blind, randomized, placebo-controlled phase I/II trial. J Gastroenterol Hepatol 1999;14:1093-1099.

24 Bousquet J, Khaltaev N, Cruz AA, Denburg J, Fokkens WJ, Togias A, et al: Allergic Rhinitis and its Impact on Asthma (ARIA) 2008 Update (in collaboration with the World Health Organization, GA(2)LEN and AllerGen). Allergy 2008;63(suppl 86):8-160.

25 Meltzer EO, Caballero F, Fromer LM, Krouse JH, Scadding G: Treatment of congestion in upper respiratory diseases. Int J Gen Med 2010;8:69-91. 
Bellussi et al:: Rhinosinusal Inflammation and High Mobility Group Box 1 Protein: A New Target for Therapy

26 Craig TJ, Sherkat A, Safaee S: Congestion and sleep impairment in allergic rhinitis. Curr Allergy Asthma Rep 2010;10:113-121.

27 Sardana N, Craig TJ: Congestion and sleep impairment in allergic rhinitis. Asian Pac J Allergy Immunol 2011; 29:297-306.

28 Lunn M, Craig T: Rhinitis and sleep. Sleep Med Rev 2011;15:293-299.

29 Salpietro C, Cuppari C, Grasso L, Tosca MA, Miraglia Del Giudice M, La Rosa M, et al: Nasal high mobility group box 1 protein in children with allergic rhinitis. Int Arch Allergy Immunol 2013;161:116-121.

30 Collins J: Prevalence of selected chronic conditions: United States, 1990-1992. Vital Health Stat 10 1997;130: $1-89$.

31 Fokkens W, Lund VJ, Mullol J, Bachert C, Alobid I, Baroody F, et al: European Position Paper on Rhinosinusitis and Nasal Polyps 2012. Rhinol Suppl 2012;3 p preceding table of contents,1-298.

32 Meltzer EO, Caballero F, Fromer LM, Krouse JH, Scadding G: Treatment of congestion in upper respiratory disease. Int J Gen Med 2010;8:69-91.

33 Passali D, Salerni L, Passali GC, Passali FM, Bellussi ML: Nasal decongestant in the treatment of chronic nasal obstruction: efficacy and safety of use. Expert Opin Drug Saf 2006;5:783-790.

34 Al Sayyad JJ, Fedorowicz Z, Alhashimi D, Jamal A: Topical nasal steroids for intermittent and persistent allergic rhinitis in children. Cochrane Database Syst Rev 2007;24:CD003163.

35 Damiani V, Camaioni A, Viti C, Schillani G, Foltran F, Scirè AS, et al: Short-term efficacy of Narivent ${ }^{\circledR}$ in the treatment of nasal congestion. Open Med Dev J 2012;4:66-72.

36 Damiani V, Camaioni A, Viti C, Schillani G, Foltran F, Scirè AS, et al: Long-term efficacy of Narivent ${ }^{\circledR}$ in the treatment of nasal congestion. Open Med Dev J 2012;4:73-79.

37 Mansi N, D’Agostino G, Scire'AS, Morpurgo G, Gregori D, Gulati A, etal: Allergic rhinitis in children: a randomized clinical trial targeted at symptoms. Indian J Otolaryngol Head Neck Surg 2014;66:386-393. 